\title{
Negative emotional processing induced by spoken scenarios modulates corticospinal excitability
}

\author{
Anna Baumert • Craig Sinclair • Colin MacLeod • \\ Geoff Hammond
}

Published online: 24 May 2011

(C) Psychonomic Society, Inc. 2011

\begin{abstract}
Emotion is assumed to facilitate the preparation of behavioral responses to environmental stimuli. In the present study, we examined whether emotional processing induced by spoken scenarios of positive and negative content, related to the self or to other people, modulates corticospinal excitability. Motor evoked potentials (MEPs) elicited by transcranial magnetic stimulation (TMS) in 20 volunteers indicated that processing of negative emotional content increased MEP amplitude, regardless of the perspective taken in the scenario. By contrast, positive emotional processing did not reliably alter MEP amplitude. These results provide the first TMS evidence that the auditory processing of emotionally negative information triggers action preparation.
\end{abstract}

Keywords TMS · Negative emotion · Positive emotion · Corticospinal excitability $\cdot$ Action preparation

A crucial function of emotion is to prepare an individual to execute behavioral responses (Frijda, 1986). Evolutionary approaches stress the importance of emotion triggering quick and effortless reactions to secure the survival of the organism (Lang, Bradley, \& Cuthbert, 1997; Lang, Davis, \& Öhman, 2000; Öhman, 1986). Several psychophysiological studies have provided evidence that visual processing of emotional content is associated with action preparation, indicated by stronger peripheral reflexes (Bonnet, Bradley,

\footnotetext{
A. Baumert $(\square)$

Department of Psychology, University of Koblenz-Landau, Fortstraße 7,

76829 Landau, Germany

e-mail: baumert@uni-landau.de

C. Sinclair $\cdot$ C. MacLeod $\cdot$ G. Hammond

Department of Psychology, University of Western Australia, Perth, Western Australia, Australia
}

Lang, \& Requin, 1995; Both, Everaerd, \& Laan, 2003), increased activity in motor areas of the brain (Bremner, Staib, Kaloupek, Southwick, Soufer \& Charney, 1999; Rauch, van der Kolk, Fisler, Alpert, Orr, Savage et al., 1996), and increased excitability of the corticospinal tract (Baumgartner, Willi, \& Jäncke, 2007; Coombes, Tandonnet, Fujiyama, Janelle, Cauraugh \& Summers, 2009; Hajcak, Molnar, George, Bolger, Koola \& Nahas, 2007; Oliveri, Babiloni, Filippi, Caltagirone, Babiloni, Cicinelli et al., 2003; Schutter, Hofman, \& van Honk, 2008; van Loon, van den Wildenberg, van Stegeren, Hajcak, \& Ridderinkhof, 2010). Specifically, studies employing transcranial magnetic stimulation (TMS) have shown that after presentation of valent but not neutral pictures, stimulation of the motor cortex with magnetic pulses results in larger motor evoked potentials (MEPs) in peripheral muscles (Coombes et al., 2009; Hajcak et al., 2007; Schutter et al., 2008; van Loon et al., 2010). These findings have been taken as evidence that emotional processing activates the central motor system, possibly in order to facilitate quick action in response to the emotioneliciting situation.

Importantly, previous TMS studies successfully demonstrating this effect have relied almost exclusively on the visual presentation of valent information to induce emotional processing. To date, only one study has found increased corticospinal motor responses to TMS following an emotional-processing manipulation that did not employ visual stimuli. Specifically, Oathes, Bruce, and Nitschke (2008) observed that such responses were larger after a worry induction, as compared to a period of rest. In contrast, Baumgartner et al. (2007) did not find evidence of increased corticospinal excitability after the presentation of emotional music alone, but they observed this only when participants were exposed to valent pictures combined with emotional music. These authors suggested that visual 
stimuli might direct attention toward external events, which may be a necessary condition for action preparation to occur at a central level. However, their results may instead be attributable to the fact that, in their study, emotionality induced by means of music alone was less pronounced than that induced using pictures or a combination of pictures and music. Therefore, it remains uncertain whether auditory processing of emotional information activates motor responses in the central nervous system or whether such activation results only from the visual processing of emotional images.

In the present study, we tested whether the auditory presentation of emotional scenarios increases the excitability of the corticospinal system, as assessed with single-pulse TMS. If emotional processing in sensory modalities other than vision also heightens action preparation, as proposed by mainstream theories of emotion (Frijda, 1986), then listening to descriptions of emotional events should increase corticospinal excitability. Such action preparation may be disproportionately evident when emotional information is processed in a self-referential manner. However, strong emotion can also be induced by observation of other people's experiences (e.g., Davis, 1996; Decety \& Jackson, 2004). Most importantly, recent findings have suggested that one's own emotional experiences and information about the emotional experiences of others may be processed in similar manners (Hennenlotter, Schroeder, Erhard, Castrop, Haslinger, Stoecker et al., 2005; Wicker et al., 2003). Thus, the modulation of corticospinal excitability when processing scenarios that describe emotional events occurring to others would further support the notion that emotional processing in general heightens action preparation.

A further issue that we wanted to explore concerns whether only negative emotional information affects corticospinal excitability, or whether this effect could also be observed with positive information. On the one hand, it could be expected that both positive and negative emotionality could increase action preparation, as both can signal the need for action. Positive emotions are related to approach tendencies, while negative emotions are related to avoidance tendencies (Coombes et al., 2009; Hajcak et al., 2007). On the other hand, it also is plausible that negative emotion may warrant the most rapid action-directed responses, and so would be disproportionately likely to trigger preparation for motor responses in the nervous system. The evolutionary basis for this contention comes from the observation that quick responses to environmental threat likely secured survival, whereas behavioral responses to positive stimuli (such as smiling faces or attractive berries) could be made more slowly and deliberatively without negative consequences, and with potential benefits (Lang et al., 1997; Öhman, 1997; Öhman \& Wiens, 2003).

Previous research using visual stimuli has provided contradictory evidence concerning whether corticospinal excitability is equally modulated by negative and by positive emotional stimuli. Employing the International Affective Picture System (Lang, Bradley, \& Cuthbert, 1999), two studies (Baumgartner et al., 2007; Hajcak et al., 2007) have observed equivalent effects with pleasant and unpleasant pictures. Another study has instead demonstrated significant effects only for unpleasant but not for pleasant pictures, as compared to neutral pictures (Coombes et al., 2009). Furthermore, in the study of Schutter et al. (2008), corticospinal excitability was modulated only by viewing fearful facial expressions and not happy facial expressions. However, because participants in this study did not rate the emotionality of the faces, it cannot be determined whether the pictures of happy faces might have been relatively mild in emotional intensity. In the present study, we compared the effects of listening to spoken descriptions of positive and negative scenarios, in order to measure the extent to which the processing of both positive and negative auditory emotional information serves to modulate excitability of the corticospinal system. Importantly, we also assessed the subjective valence of the scenarios, in order to compare the emotional intensity of negative and positive scenarios, and in order to determine whether variation in subjective valence was associated with variability in the modulation of corticospinal excitability.

\section{Method}

\section{Participants}

A total of 23 Caucasian, Asian, and African persons (13 female) agreed to participate in this TMS experiment. They gave their written consent after they were fully informed about the procedure by means of an information sheet approved by the ethics committee of the University of Western Australia. Their ages ranged from 16 to 35 years $(M=21.23, S D=4.9)$. All participants indicated that they were right-handed on a dichotomous forced choice scale. Volunteers were excluded if they reported any contraindication to TMS. As a safety precaution, participants who were pregnant, had any metal implants in their head, were currently taking psychoactive medication, or had a history of fainting were not tested.

\section{Apparatus}

Stimuli A set of 96 scenarios was employed in the TMS experiment. Two versions of each scenario were prepared. One version used the second-person pronoun "you," such that events were described as though being experienced by the listener (self perspective), while the other version substituted this pronoun with references to a third party unrelated to the listener (other perspective). Of the 
scenarios, 32 described positive events, 32 described negative events, and 32 described neutral events, all similar to events reported in everyday media. Examples of each type of scenario are provided in Table 1.

A digital audio recording was made of both versions of each scenario. Half of the scenarios were recorded by a male native English speaker, and the other half by a female native English speaker (scenario valence and perspective were fully balanced across speakers). All recordings were peak normalized to $0 \mathrm{~dB}$. For all scenarios, the sample size was 16 bits and the sampling rate was $44 \mathrm{kHz}$. Duration of the scenarios varied between 5 and $17 \mathrm{~s}(M=9.19, S D=2.28)$. The mean length of positive scenarios was $8.16 \mathrm{~s}(S D=2.01)$, the mean length of negative scenarios was $9.92 \mathrm{~s}(S D=2.42)$, and the mean length of neutral scenarios was $9.50 \mathrm{~s}(S D=2.02)$,

In order to ensure that passage types differed in emotional valence as intended, in both their selfperspective and other-perspective formats, a rating approach was adopted. A group of 10 raters (all native English speakers, 7 female; age range: $17-21$ years, $M=$ $18.60, S D=1.26$ ) were presented with the recorded scenarios, and reported how positive or negative they judged each to be on a nine-point response scale from -4 (very negative) to +4 (very positive). Half of the raters listened to scenarios in their other-perspective format and were instructed to imagine that the described situation happened to the person identified in the passage. The remaining participants listened to scenarios in their selfperspective format and were instructed to imagine the described situation happening to themselves. The means and standard deviations of the valence ratings are displayed in Table 1 for the different types of scenarios. We conducted a 2 (perspective: self/other) $\times 3$ (scenario valence: negative, positive, neutral) ANOVA with scenario valence as a repeated measure. ${ }^{1}$ There was a significant main effect of scenario valence, $F(2,7)=123.82, p<.001$, $\eta_{\mathrm{p}}^{2}=.97$ : Negative scenarios were perceived as significantly more negative $(M=-2.69, S D=0.32)$ than neutral scenarios $(M=0.24, S D=0.34), t(9)=-16.24, p<.001$, and positive scenarios were perceived as significantly more positive $(M=$ 2.21, $S D=0.64)$ than neutral scenarios, $t(9)=14.79, p<$ .001. Neither the main effect of perspective nor the Perspective X Scenario Valence interaction was significant, all $F$ s $<1$. Hence, scenarios differed in their perceived valence as intended. In the main experiment, Valence and Perspective were both retained as factors. Despite scenarios being rated as similarly valent independent of the perspective

\footnotetext{
${ }^{1}$ As explained below (see the Data Analyses section), a multivariate approach was used for all general linear model within-subjects comparisons. In our prestudy, the sphericity assumption was violated (Greenhouse-Geisser $\varepsilon=.59$ for the main effect of scenario valence).
}

they were presented in, one goal of the present study was to explore whether they would also be processed in identical manners. Particularly, emotional processing triggered in the self-perspective condition may have a disproportionate impact on the preparation of motor responses.

Transcranial magnetic stimulation TMS was delivered by a Magstim $200^{2}$ BiStim system (Magstim Co., Whitland, U.K.) and a figure-8-shaped coil (90 $\mathrm{mm}$ in diameter) placed over the scalp of the left hemisphere, oriented at $45^{\circ}$ to the midsagittal line, with the handle facing backward, to induce current in a posterior-to-anterior direction. The optimal stimulation site was identified as the scalp site where the largest mean MEP amplitude was measured in the right first dorsal interosseous (FDI) muscle in a sequence of five moderately suprathreshold pulses. An individual's resting motor threshold was determined as the lowest stimulator intensity at which at least three out of five pulses elicited MEPs greater than $50 \mu \mathrm{V}$ in the relaxed right FDI muscle. The TMS was set at the intensity required to elicit a 1-mV (peakto-peak) MEP at rest. This intensity was used during the experiment. The mean intensity was $56.8 \%(S D=8.0 \%)$ of maximum stimulator output.

$M E P$ recording and measurement Electromyographic (EMG) activity in the focal muscle was measured by means of two surface electrodes fixed in a standard belly-tendon montage. A ground electrode was placed over the lateral posterior tubercle of the radius. Before electrodes were attached, the skin was cleaned with an abrasive gel, and a conducting paste was applied. Signals were amplified, bandpass filtered $(10-1000 \mathrm{~Hz})$, and digitized at $4000 \mathrm{~Hz}$ with a 14-bit analog-to-digital converter. The peak-to-peak MEP amplitude on each trial was calculated as the maximum EMG signal minus the minimum EMG signal, in the EMG swathe 10-50 ms after the TMS pulse. This captures the MEP, while avoiding coding the stimulus artifact as part of the biological response.

Any trial on which the EMG signal measured in the preTMS interval (50 ms prior to TMS delivery) was greater than $50 \mu \mathrm{V}$ was removed from the analysis (between 0 and 17 trials per participant; 78 trials in total across participants). This is important, in order to exclude the possibility that MEPs could be confounded with participants getting ready to move their hand for ratings. Trials removed varied unsystematically across Valence $\times$ Perspective conditions, and no participant had less than 3 valid TMS trials per condition.

Procedure

Participants were seated comfortably, with their chins on a chinrest, in front of a computer screen. Their right arm was 
Table 1 Examples of negative, neutral, and positive scenarios in the self- and other-perspective formats, as well as means and standard deviations of subjective ratings in the pilot study

\begin{tabular}{|c|c|c|c|c|}
\hline \multirow[t]{2}{*}{ Type of Scenario } & & \multirow[t]{2}{*}{ Example Scenario } & \multicolumn{2}{|c|}{ Subjective Ratings } \\
\hline & & & $M$ & $S D$ \\
\hline \multirow[t]{2}{*}{ Negative } & Self & $\begin{array}{l}\text { You are left without food and water after a hurricane } \\
\text { ravaged the country. Severe floods are expected to } \\
\text { worsen the situation dramatically for the victims. }\end{array}$ & -2.60 & 0.34 \\
\hline & Other & $\begin{array}{l}\text { Many people are left without food and water after a } \\
\text { hurricane ravaged the country. Severe floods are expected } \\
\text { to worsen the situation dramatically for the victims. }\end{array}$ & -2.78 & 0.31 \\
\hline \multirow[t]{2}{*}{ Neutral } & Self & $\begin{array}{l}\text { You like dogs which are a domesticated subspecies of the wolf. } \\
\text { This species has developed into hundreds of varied breeds. }\end{array}$ & 0.28 & 0.34 \\
\hline & Other & $\begin{array}{l}\text { Many people like dogs which are a domesticated subspecies of } \\
\text { the wolf. This species has developed into hundreds of varied breeds. }\end{array}$ & 0.21 & 0.38 \\
\hline \multirow[t]{2}{*}{ Positive } & Self & $\begin{array}{l}\text { In the vacation, you are heading out for your favorite activity. } \\
\text { You enjoy the holidays very much. }\end{array}$ & 2.28 & 0.47 \\
\hline & Other & $\begin{array}{l}\text { In the vacation, employees of a large business are heading out } \\
\text { for their favorite activity. They enjoy the holidays very much. }\end{array}$ & 2.14 & 0.83 \\
\hline
\end{tabular}

$N=10$. Subjective ratings are on a nine-point response scale from -4 (very negative) to +4 (very positive)

placed on the arm rest of the chair and the hand on the desk in front of them, on top of a computer mouse used to rate the scenarios. ${ }^{2}$ Participants maintained this posture during the preliminary measurement of their resting motor threshold and during the main experiment.

Participants listened to the recorded scenarios via earphones. They were instructed to rate each scenario regarding its pleasantness or unpleasantness on a sevenpoint response scale from -3 (very negative) to +3 (very positive) using the mouse. After the end of each scenario, and after delivery of the TMS pulse, a visual response scale appeared on the otherwise black screen together with the instruction to "Respond now." Participants rated the scenario, and the next scenario started with a delay of 2,000-6,000 ms, depending on the Magstim arming time for the next trial.

Each participant listened to and rated all 96 scenarios, 48 in the self perspective, and 48 in the other perspective. Across participants, we counterbalanced which specific scenario was presented in which perspective. Scenarios were presented in 16 blocks of six scenarios, with each of the six scenarios in each block adopting the same perspective. At the beginning of each block, the cue "yourself" or "someone else" appeared on the computer screen, to indicate the perspective that would be employed within that block. Participants were asked to imagine the described situation happening either to themselves (self perspective) or to another person (other perspective),

\footnotetext{
${ }^{2}$ In case participants had high resting EMG activity, they were asked to rest their hand flat on the table during the trial and only to move it to employ the mouse for the ratings.
}

depending on this cue. Participants switched perspectives after each block, and the order of blocks (i.e., self, other, self, etc., or other, self, other, etc.) was counterbalanced across participants. Scenarios were randomly assigned to blocks, depending on the perspective they were presented in for an individual participant. The order of scenarios within a block was also random. Three of the scenarios in each block were followed by a single TMS pulse, resulting in a total of 48 TMS trials per participant. Pulses were assigned to scenarios at random, the only restriction being that for each participant equal numbers of negative, neutral, and positive scenarios were followed by a pulse. Consequently, for each participant, there were 8 TMS trials per Valence $\times$ Perspective condition. At the end of the experiment, participants were thanked and fully debriefed.

\section{Data analyses}

To analyze subjective ratings and MEPs as dependent measures, mean scores were calculated separately for trials with negative, positive, and neutral scenarios presented in the self- and other-perspective formats. To correct for outliers, MEPs smaller than $50 \mu \mathrm{V}$ were omitted before means were calculated (across participants, five trials were excluded).

Separate 2 (perspective: self/other) $\times 3$ (scenario valence: negative/positive/neutral) ANOVAs were calculated with perspective and valence as repeated measures. A multivariate approach was used for all general linear model within-subjects comparisons. Importantly, the multivariate approach in repeated measures analyses does not require sphericity, and it is recommended over the univariate approach because of higher power in cases of substantial 
violation of sphericity and sample sizes larger than the number of levels of repeated measures (Algina \& Keselman, 1997; Maxwell \& Delaney, 2004), as encountered in our study. ${ }^{3}$

To analyze the association between subjective valence ratings and MEPs, we estimated a hierarchical linear model with MEPs as the dependent measure and included the Deviation of a Participant's Rating From His or Her Mean Rating for each scenario as a fixed factor, as well as the random intercepts (Norusis, 2005). This analysis accounts for the fact that scenarios are nested within persons.

\section{Results}

The data from 3 participants were omitted from the final analyses because they had excessive resting EMG activity or because they misinterpreted the instructions. The means and standard deviations for the subjective ratings and MEPs of the remaining participants are given in Table 2, separately for negative, neutral, and positive scenarios presented in the selfperspective and other-perspective formats.

Effects of scenario valence and perspective on subjective ratings

Analysis of the subjective ratings revealed a significant main effect of scenario valence, $F(2,18)=164.58, p<.001, \eta_{\mathrm{p}}^{2}=$ .95 (see Fig. 1). Consistent with the rating data collected in our pilot study, negative scenarios were rated as more negative $(M=-2.26, S D=0.42)$ than neutral ones $(M=$ $0.49, S D=0.45), t(19)=-16.95, p<.001$, and positive scenarios were rated as more positive $(M=1.93, S D=0.63)$ than neutral ones, $t(19)=13.30, p<.0001$. In contrast to the ratings obtained in the pilot study, however, there was now a significant main effect of perspective, $F(1,19)=7.30, p=$ $.01, \eta_{\mathrm{p}}^{2}=.28$, as well as a significant Scenario Valence $\times$ Perspective interaction, $F(2,18)=8.04, p<.01, \eta_{\mathrm{p}}^{2}=.47$. Positive scenarios were rated significantly more positive in the self perspective $(M=2.16, S D=0.68)$ than in the other perspective $(M=1.71, S D=0.65), t(19)=4.72, p<.001$, whereas ratings of negative scenarios did not differ between perspectives, $t(19)=-1.12, p=.28$. Ratings of neutral scenarios also did not differ between perspectives, $t(19)=$ $1.41, p=.17$.

It is important to note, however, that the simple main effect of scenario valence remained statistically significant for passages in both the self-perspective format, $F(2,18)=$

\footnotetext{
$\overline{3}$ In our analyses, the sphericity assumption was violated (in the analysis of subjective ratings, Greenhouse-Geisser $\varepsilon=.63$ for the main effect of scenario valence; in the analysis of $M E P \mathrm{~s}$, GreenhouseGeisser $\varepsilon=.68$ and .83 for the main effect of scenario valence and the Perspective $\times$ Valence interaction, respectively).
}

Table 2 Means and standard deviations of subjective ratings and MEPs (in microvolts) for negative, neutral, and positive scenarios in the self- and other-perspective formats in the TMS experiment

\begin{tabular}{|c|c|c|c|c|c|}
\hline \multicolumn{2}{|c|}{ Type of Scenario } & \multicolumn{2}{|c|}{ Subjective Ratings } & \multicolumn{2}{|l|}{ MEPs } \\
\hline & & $M$ & $S D$ & $M$ & $S D$ \\
\hline \multirow[t]{2}{*}{ Negative } & Self & -2.31 & 0.48 & 2,023 & 1,325 \\
\hline & Other & -2.22 & 0.44 & 1,945 & 1,410 \\
\hline \multirow[t]{2}{*}{ Neutral } & Self & 0.59 & 0.58 & 1,816 & 1325 \\
\hline & Other & 0.39 & 0.50 & 1,691 & 1,187 \\
\hline \multirow[t]{2}{*}{ Positive } & Self & 2.16 & 0.68 & 1,957 & 1,261 \\
\hline & Other & 1.71 & 0.65 & 1,658 & 1,268 \\
\hline
\end{tabular}

$N=20$. Subjective ratings are on a seven-point response scale from -3 (very negative) to +3 (very positive)

161.37, $p<.001, \eta^{2} \mathrm{p}=.95$, and the other-perspective format, $F(2,18)=151.51, p<.001, \eta_{\mathrm{p}}^{2}=.94$. Therefore, despite positive scenarios being rated as less positive in the other-perspective than in the self-perspective condition, they were still rated as significantly more positive than neutral scenarios in either the other-perspective condition, $t(19)=9.55, p<.001$, or the self-perspective condition, $t(19)=12.88, p<.001$.

To determine whether the intensity of emotionality differed for negative and positive scenarios, we compared the absolute values of rating scores for these categories of scenarios, separately for the self-perspective and otherperspective formats. In the self-perspective condition, there was no significant difference between the absolute values of subjective valence ratings of negative and positive scenarios, $t(19)=-1.55, p=.14, d=0.25$. By contrast, in the other-perspective condition, the absolute values of the subjective valence ratings differed significantly, $t(19)=-4.74, p<.001, d=1.89$, with negative scenarios being rated as more emotionally intense than positive scenarios (see Table 2).

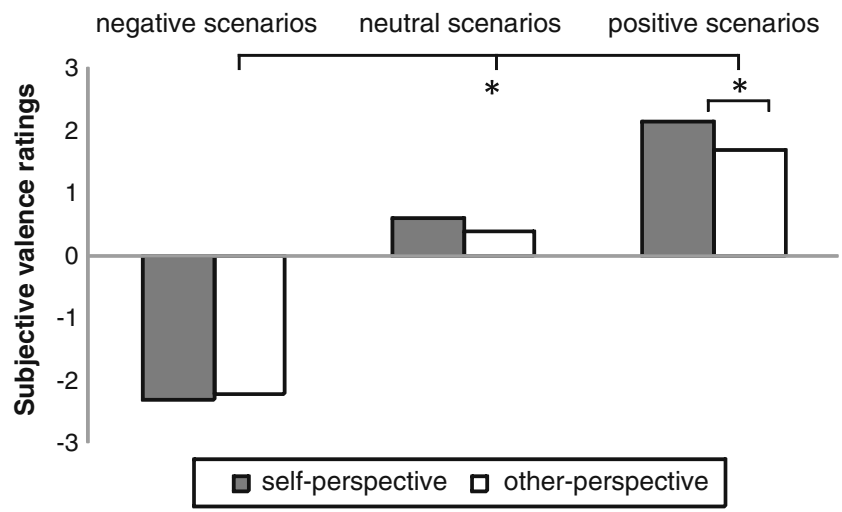

Fig. 1 Subjective valence ratings as a function of valence condition (negative, neutral, or positive scenarios) and perspective (self or other). ${ }^{*} p<.05$ 
Effects of scenario valence and perspective on MEPs

The analysis of MEP amplitude revealed a significant main effect of scenario valence, $F(2,18)=4.07, p=.04, \eta_{\mathrm{p}}^{2}=$ .31 (see Fig. 2): MEPs elicited after negative scenarios were significantly larger $(M=1,984, S D=1,309)$ than those elicited after neutral scenarios $(M=1,753, S D=1,187), t$ $(19)=2.93, p<.01$. Descriptively, this pattern was evidenced in 75\% (15/20) of the participants. MEPs elicited after positive scenarios were not significantly larger $(M=$ $1,808, S D=1,234)$ than those elicited after neutral scenarios, $t<1$. Also, there was no significant difference between MEPs elicited after negative and after positive scenarios, $t(19)=1.05, p=.31$. Descriptively, for $40 \%(8 /$ 20) of the participants, MEPs elicited after positive scenarios were smaller than those after neutral and negative scenarios. For $35 \%(7 / 20)$, MEPs elicited after positive scenarios were intermediate.

No other significant effects emerged from this analysis. It is of particular importance to note that the above-described main effect of scenario valence was not significantly modified by perspective in a two-way interaction $(F<1)$.

To exclude the possibility that the observed effects could be attributable to baseline differences in MEPs prior to TMS, we computed the mean pre-TMS EMG activity recorded from $50 \mathrm{~ms}$ prior to TMS, through until TMS onset. A 2 (perspective: self/other) $\times 3$ (scenario valence: negative/positive/neutral) ANOVA, with Perspective and Valence as repeated measures factors, showed that neither the main effect of valence, $F(2,18)=1.60, p=.23$, $\eta_{\mathrm{p}}^{2}=.15$, the main effect of perspective, $F(1,19)=1.31$, $p=.27, \eta_{\mathrm{p}}^{2}=.06$, nor the Perspective $\times$ Valence interaction, $F(2,18)<1$, was significant. Hence, it can be concluded that baseline EMG activity did not differ across scenario conditions.

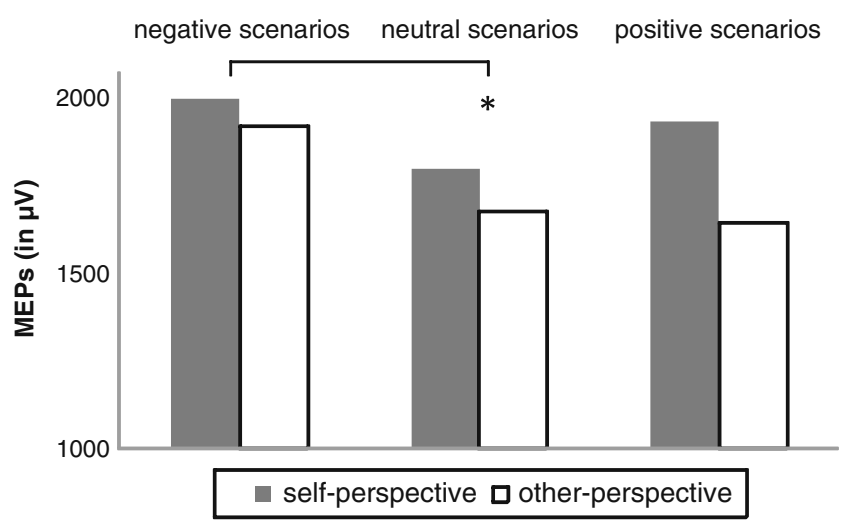

Fig. 2 Motor evoked potentials (in microvolts) as a function of valence condition (negative, neutral, or positive scenarios) and perspective (self or other). ${ }^{*} p<.05$
Association between subjective valence ratings and MEPs

We explored the correlations between subjective valence ratings and MEP amplitudes in order to complement our main analyses. Specifically, we were interested in whether variance within participants in the ratings given to the scenarios was related to variance in the MEPs they evidenced after exposure to these scenarios. Therefore, we estimated a hierarchical linear model including the Deviation of a Participant's Rating From His or Her Mean Rating for each scenario as a fixed factor, as well as the random intercepts (Norusis, 2005). ${ }^{4}$ The correlation between within-subjects variation in valence ratings and in MEPs, across scenarios, showed a strong trend toward significance, $F(1,853.07)=$ $3.46, p=.06$. The parameter estimate of the average slope was $-47.17(S E=25.35)$. This suggests that there was a tendency toward larger MEPs being elicited by TMS pulses that followed scenarios that a participant rated as more negative, relative to TMS pulses delivered after scenarios that the participant rated as more positive.

\section{Discussion}

A prominent theoretical view is that emotions drive behavior by preparing the organism for quick and effortless reactions (Frijda, 1986). The present study adds to the body of empirical evidence that the processing of emotionally negative information can serve to elicit action preparation at the level of the central nervous system. Previous studies have consistently shown that the presentation of negative emotional pictures increases MEPs in peripheral muscles when evoked by TMS applied to the motor cortex (Coombes et al., 2009; Hajcak et al., 2007; Schutter et al., 2008; van Loon et al., 2010). We found similar effects using auditory, rather than visual, processing. Exposure to spoken scenarios describing emotionally negative events modulated corticospinal excitability. Thus, complementing previous studies, our results indicate that action preparation due to negative emotional processing is not limited to the visual channel (cf. Oathes et al., 2008).

This finding is in line with evidence in affective neuroscience showing that specific areas of the brain, such as the anterior cingulate cortex and the hippocampus, are involved in emotional processing, independent of the sensory channel through which it is evoked (Davidson, Pizzagalli, Nitschke, \& Kalin, 2003). Importantly, there is evidence that emotional processing is closely linked to behavioral responses independent of sensory modality. For example, the amygdala, receiving highly processed sensory

\footnotetext{
${ }^{4}$ A model including random slopes showed that their variance was nonsignificant.
} 
information from all modalities, is involved in the acquisition and expression of fear (Lang et al., 2000). Additionally, social psychological research has indicated that the processing of both affective words (e.g., "hostile" vs. "polite") and valent pictures can shape behavior by priming related behavioral scripts (e.g., Bargh, Chen, \& Burrows, 1996). Thus, our findings add to the body of evidence suggesting that the emotion and motor systems are integrated at a fundamental level (cf. Coombes et al., 2009). Specifically, together with prior TMS studies, our results suggest that negative emotional processing swiftly prepares the organism for behavioral action.

Furthermore, our study found evidence that exposure to emotionally negative scenarios served to modulate corticospinal excitability independent of the perspective that listeners take toward the described events. This result is in accordance with the finding that humans process information concerning the emotional experiences of others in a way similar to their own emotional experiences (Decety \& Jackson, 2004; Hennenlotter et al., 2005; Wicker et al., 2003). Apparently, negative emotional processing induced by spoken descriptions of one's own, or of other people's, emotional experiences prepares the organism to react. From an evolutionary perspective, this mirror-like mechanism, through which the processing of emotional information concerning others reflects that of emotional information concerning oneself, seems likely to be adaptive, given that the negative experiences of others often also imply a threat to oneself.

Previous research has been inconclusive concerning whether only negative emotions or both negative and positive emotions trigger action preparation (Baumgartner et al., 2007; Coombes et al., 2009; Hajcak et al., 2007; Schutter et al., 2008; van Loon et al., 2010). In accordance with the findings of Schutter et al. (2008), and in contrast to those of Baumgartner et al. (2007) and Hajcak et al. (2007), we found that only negative emotional processing modulated corticospinal excitability. Similar to the pattern of results obtained by Coombes et al. (2009), in our study positive emotional processing was intermediate: On the one hand, corticospinal excitability was not significantly lower after the processing of positive scenarios than after the processing of negative scenarios. On the other hand, the processing of positive scenarios did not lead to significantly increased corticospinal excitability, as compared with the processing of neutral scenarios. Thus, from our results, it cannot be concluded that positive emotional processing triggers action preparation at a central level. This was further supported by our analysis of the association between the subjective valence ratings of scenarios and the MEPs elicited after the processing of these scenarios. This analysis revealed that corticospinal excitability tended to be greater after processing scenarios rated as subjectively more negative than after scenarios rated as subjectively more positive.
Importantly, in the present study this result cannot be attributed to the positive stimuli having been less emotionally intense than the negative stimuli. The subjective valence ratings showed that, in the self-perspective format, positive and negative scenarios differed from neutrality to an equivalent degree. Only in the other-perspective format were positive scenarios rated as less emotionally intense than negative scenarios. However, this interaction of perspective and scenario valence was not reflected in the MEPs. If emotional intensity rather than the valence of scenarios was responsible for modulating corticospinal excitability, then processing positive scenarios in the self-perspective format should have significantly increased MEP amplitudes, even if this was not the case when the scenarios were presented in the other-perspective format.

Why, then, might it be that some previous TMS studies appeared to show that the processing of positive stimuli also can modulate corticospinal excitability? One possibility is suggested by the observation that studies finding such an effect typically have blocked stimuli by emotional valence during presentation, rather than presenting differentially valenced stimuli in a random order. In blocked presentations of valent stimuli, TMS is repeatedly applied while either positive or negative emotional processing continues across successive stimuli that all share the same emotional tone. In contrast, random presentation means that the application of TMS occurred immediately after a single description of a particular valence was listened to or right after a single valent picture was presented. This invites speculation that perhaps the processing of both positive and negative information might modulate corticospinal excitability, but that each effect may operate across a differing time course. Whereas negative emotional processing appears to trigger action preparation immediately, positive emotional processing may take longer to do so. Consistent with this possibility, Schutter et al. (2008) found modulation of corticospinal excitability after presentation of fearful faces (but not happy faces) as little as $300 \mathrm{~ms}$ after stimulus onset. By contrast, for positive stimuli, modulation of corticospinal excitability has been found only during longer intervals of continued positive emotional processing (e.g., presentation of positive pictures and positive music for $70 \mathrm{~s}$ in Baumgartner et al., 2007; presentation of more than 40 positive pictures for $3 \mathrm{~s}$ each in Hajcak et al., 2007; presentation of 240 positive pictures for $1 \mathrm{~s}$ each in van Loon et al., 2010). In our study, the duration of emotional processing was intermediate; the length of the scenarios was on average $9 \mathrm{~s}$. Thus, it remains uncertain whether continued processing of positive emotional information for durations in excess of $9 \mathrm{~s}$ might come to modulate corticospinal excitability. Further research could address this issue by systematically varying the time lag between the onset of emotional information processing and the 
application of TMS. Complementing the approach of van Loon et al., (2010) the presentation of valent stimuli could be structured to introduce a range of intervals between exposure to emotional information and delivery of TMS, in order to test whether positive stimuli can modulate corticospinal excitability to the same degree that negative stimuli do, but less rapidly. This explanation would be consistent with the evolutionary contention that rapid reactions in the face of threat may best secure survival of the organism, whereas appetitive reactions bring greater benefits by being persistent rather than by being quick (Lang et al., 1997; Öhman, 1997; Öhman \& Wiens, 2003).

An alternative reason why positive stimuli may have proven less consistently effective in modulating corticospinal excitability has been proposed by Coombes et al. (2009). They argued that this might reflect the fact that previous work has predominantly recorded MEPs from extensor muscles rather than flexor muscles. Specifically, Coombes et al. suggested that MEPs in muscles involved in extension movements (i.e., away from the body, implying avoidance) might reveal action preparation due to negative (rather than positive) emotional processing because of the congruency of the motivational and motor systems. Our findings do not support this account, however, because we found significant modulating effects of negative, but not of positive, scenarios as compared to neutral scenarios, despite measuring MEPs from a flexor muscle (i.e., FDI). Nevertheless, future research might profit from more systematically evaluating the impact of emotional processing on action preparation involving approach and avoidance motivation, in a manner that contrasts MEPs recorded from such differing muscle groups.

\section{Limitations}

When drawing conclusions from our findings, several limitations should be taken into account, which could be overcome in future research. First, our study provides evidence that action preparation due to negative emotional processing is not limited to the visual channel, but can also occur due to auditory processing of negative spoken scenarios. It should be noted, though, that our participants were instructed to imagine the described situation. Thus, it remains to be tested whether mental imagery is a necessary condition for the auditory processing of negative information to modulate corticospinal excitability (cf. Baumgartner et al., 2007).

Second, in our study possible carryover effects from one scenario to the next cannot be excluded. So far, there has been little systematic research on the time course of the affective modulation of corticospinal activity. As stated above, the exact onset of a potentially slower effect of positive (as compared to negative) emotional processing remains to be tested. Similarly, it is unknown how long corticospinal excitability remains enhanced after emotional processing has terminated. The time lag between scenarios was at minimum 2,000-6,000 ms in our study, and so it is possible that MEPs elicited after a scenario might have been influenced by the valence of the previous scenario. Such carryover could be increased if participants continued to ruminate about previous scenarios. However, because of the randomized order of scenarios in our study, any such carryover effects would not have been systematically confounded with the effect of scenario valence, and so could not have artifactually produced the observed pattern of results. Rather, they would have resulted only in error variances that would have contributed to the underestimation of effects.

Third, future studies designed to illuminate the precise temporal parameters of the corticospinal response to emotional information processing could more exactly match the length of positive, negative, and neutral scenarios, which in the present study varied slightly. Moreover, to ensure maximum emotional intensity, regardless of whether passages are presented in the self-perspective or the other-perspective format, it might be necessary to generate more emotionally intense positive scenarios. Whereas in our pilot study we found no difference in the subjective valences of scenarios due to the different perspectives, participants in our main experiment rated positive scenarios in the other-perspective format to be less positive, and thus less emotionally intense, than when they were presented in the self-perspective format. Although this difference in emotional intensity due to scenario perspective was not reflected in modulated corticospinal excitability, it would be desirable to employ stimulus materials in future research that would yield equivalent emotional ratings, regardless of the perspective in which they were presented.

Finally, future research could complement our present work by investigating whether individual differences shape how emotional stimuli are processed. For example, trait empathy (Davis, 1996) may influence how intensely other people's emotional experiences are felt. Hence, trait empathy might determine how strongly processing of others' emotional experiences prepares an individual to act, by modulating his or her corticospinal excitability. Some initial TMS studies already have considered individual differences in empathy and related constructs when investigating the processing of observed pain in others (e.g., Avenanti, Minio-Paluello, Bufalari, \& Aglioti, 2009).

For the moment, the results of the present study support earlier theorizing that emotion provides a driving motivation for behavior, preparing the individual to react adaptively to cues that elicit emotional states (Frijda, 1986; Öhman, 1986, 1997). The processing of emotionally negative, auditorily presented scenarios was found to elicit a significant increase in corticospinal excitability. In addition to providing evidence that the processing of emotionally negative information triggers action preparation, 
our findings also demonstrate that this effect is not restricted to the visual modality and does not depend on the information being processed in a self-referential manner. We believe that future extensions of this research approach promise to yield further insights into the relationship between the brain systems that govern emotional experience and those that control action, which is likely to have been shaped by our evolutionary heritage.

Author Note We thank Mario Gollwitzer and Jürgen Baumert for important advice regarding the data analysis. We also thank Mark Boyes and Clare Mein for the recordings of scenarios.

\section{References}

Algina, J., \& Keselman, H. J. (1997). Detecting repeated measures effects with univariate and multivariate statistics. Psychological Methods, 2, 208-218.

Avenanti, A., Minio-Paluello, I., Bufalari, I., \& Aglioti, S. M. (2009). The pain of a model in the personality of an onlooker: influence of state-reactivity and personality traits on embodied empathy for pain. Neuroimage, 44, 275-283.

Bargh, J. A., Chen, M., \& Burrows, L. (1996). Automaticity of social behavior: direct effects of trait construct and stereotype activation in action. Journal of Personality and Social Psychology, 71, 230-244.

Baumgartner, T., Willi, M., \& Jäncke, L. (2007). Modulation of corticospinal activity by strong emotions evoked by pictures and classical music: a transcranial magnetic stimulation study. Neuroreport, 18, 261-265.

Bonnet, M., Bradley, M. M., Lang, P. J., \& Requin, J. (1995). Modulation of spinal reflexes: arousal, pleasure, action. Psychophysiology, 32, 367-372.

Both, S., Everaerd, W., \& Laan, E. (2003). Modulation of spinal reflexes by aversive and sexually appetitive stimuli. Psychophysiology, 40, 174-183.

Bremner, J. D., Staib, L. H., Kaloupek, D., Southwick, S. M., Soufer, R., \& Charney, D. S. (1999). Neural correlates of exposure to traumatic pictures and sound in Vietnam combat veterans with and without posttraumatic stress disorder: a positron emission tomography study. Biological Psychiatry, 45, 806-816.

Coombes, S. A., Tandonnet, C., Fujiyama, H., Janelle, C. M., Cauraugh, J. H., \& Summers, J. J. (2009). Emotion and motor preparation: a transcaranial magnetic stimulation study of corticospinal motor tract excitability. Cognitive, Affective \& Behavioral Neuroscience, 9, 380-388.

Davidson, R. J., Pizzagalli, D., Nitschke, J. B., \& Kalin, N. H. (2003). Parsing the subcomponents of emotion and disorders of emotion: Perspectives from affective neuroscience. In R. J. Davidson, K. R. Scherer, \& H. H. Goldsmith (Eds.), Handbook of affective sciences (pp. 8-24). Oxford: Oxford University Press.

Davis, M. H. (1996). Empathy: A social psychological approach. Madison: Westview Press.

Decety, J., \& Jackson, P. L. (2004). The functional architecture of human empathy. Behavioral and Cognitive Neuroscience Reviews, 3, 71100 .

Frijda, N. H. (1986). The emotions. Cambridge: Cambridge University Press.
Hajcak, G., Molnar, C., George, M. S., Bolger, K., Koola, J., \& Nahas, Z. (2007). Emotion facilitates action: a transcranial magnetic stimulation study of motor cortex excitability during picture viewing. Psychophysiology, 44, 91-97.

Hennenlotter, A., Schroeder, T. U., Erhard, P., Castrop, F., Haslinger, B., Stoecker, D., et al. (2005). A common neural basis for receptive and expressive communication of pleasant facial affect. Neuroimage, 26, 581-591.

Lang, P. J., Bradley, M. M., \& Cuthbert, B. N. (1997). Motivated attention: Affect, activation and action. In P. J. Lang, R. F. Simons, \& M. F. Balaban (Eds.), Attention and orienting: Sensory and motivational processes (pp. 97-135). Hillsdale: Erlbaum.

Lang, P. J., Bradley, M. M., \& Cuthbert, B. N. (1999). International affective picture system: Instruction manual and affective ratings (Tech. Rep. A-4). Gainsville: University of Florida, Center for Research in Psychophysiology.

Lang, P. J., Davis, M., \& Öhman, A. (2000). Fear and anxiety: animal models and human cognitive psychophysiology. Journal of Affective Disorders, 61, 137-159.

Maxwell, S. E., \& Delaney, H. D. (2004). Designing experiments and analyzing data: A model comparison perspective. Mahwah: Erlbaum.

Norusis, M. (2005). SPSS 14.0 advanced statistical procedures companion. Upper Saddle River: Prentice Hall.

Oathes, D. J., Bruce, J. M., \& Nitschke, J. B. (2008). Worry facilitates corticospinal motor response to transcranial magnetic stimulation. Depression and Anxiety, 25, 969-976. doi:10.1002/da.20445

Öhman, A. (1986). Face the beast and fear the face: animal and social fears as prototypes for evolutionary analyses of emotion. Psychophysiology, 23, 123-145.

Öhman, A. (1997). As fast as the blink of an eye: Evolutionary preparedness for preattentive processing of threat. In P. J. Lang, R. F. Simons, \& M. F. Balaban (Eds.), Attention and orienting: Sensory and motivational processes (pp. 97-135). Hillsdale: Erlbaum.

Öhman, A., \& Wiens, S. (2003). On the automaticity of autonomic responses in emotion: An evolutionary perspective. In R. J. Davidson, K. R. Scherer, \& H. H. Goldsmith (Eds.), Handbook of affective sciences (pp. 256-275). New York: Oxford University Press.

Oliveri, M., Babiloni, C., Filippi, M. M., Caltagirone, C., Babiloni, F., Cicinelli, P., et al. (2003). Influence of the supplementary motor area on primary motor cortex excitability during movements triggered by neutral or emotionally unpleasant visual cues. Experimental Brain Research, 149, 214-221.

Rauch, S. L., van der Kolk, B. A., Fisler, R. E., Alpert, N. M., Orr, S. P., Savage, C. R., et al. (1996). A symptom provocation study of posttraumatic stress disorder using positron emission tomography and script-driven imagery. Archives of General Psychiatry, 53, 380-387.

Schutter, D. J. L. G., Hofman, D., \& van Honk, J. (2008). Fearful faces selectively increase corticospinal motor tract excitability: a transcranial magnetic stimulation study. Psychophysiology, 45, 345-348. doi:10.1111/j.1469-8986.2007.00635.x

van Loon, A. M., van den Wildenberg, W. P. M., van Stegeren, A. H., Hajcak, G., \& Ridderinkhof, K. R. (2010). Emotional stimuli modulate readiness for action: a transcranial magnetic stimulation study. Cognitive, Affective \& Behavioral Neuroscience, 10, 174-181.

Wicker, B., Keysers, C., Plailly, J., Royet, J.-P., Gallese, V., \& Rizzolatti, G. (2003). Both of us disgusted in $m y$ insula: the common neural basis of seeing and feeling disgust. Neuron, 40, 655-664. doi:10.1016/S0896-6273(03)00679-2 